\title{
The Future for UV Spectroscopy of the ISM at High Resolution
}

\author{
Edward B. Jenkins \\ Princeton University Observatory \\ Princeton, NJ 08544-1001 \\ $U S A$
}

\begin{abstract}
In the post-HST era, advanced missions for performing UV spectroscopy must excel in many ways. We must not overlook the importance of high wavelength resolution for investigating absorption lines from gas systems in the Galaxy and beyond. We consider here some basic principles on this topic, both scientific and technical.
\end{abstract}

\section{Fundamental Parameters}

Over recent decades, UV astronomy has prospered. However, HST will not last forever, which means that we must prepare for a more advanced facility - one that can allow us to travel well beyond our past accomplishments.

There are a number of figures of merit that will influence an advanced mission's capability to perform point-source spectroscopy. First, the light-gathering power is defined by an effective area $A_{\text {eff }}$, which is the product of the unobscured area of the telescope's aperture, the quantum efficiency of the detector, and the efficiency of the optics. Next, there is the multiplexing factor $M$, which is the number of independent spectral channels that can be recorded simultaneously: The product $A_{\text {eff }} M$ determines how rapidly information can be gathered from a source of a given brightness. A central theme of our discussion will be the capability to deliver a high wavelength resolving power $R \equiv \lambda / \Delta \lambda$. This property, along with the signal-to-noise ratio $(S / N)$, governs the quality of the recorded information. The wavelength band - not a real figure of merit - defines what scientific problems we can approach for a system at a given redshift $z$.

Jenkins (1999) has summarized the advances in $A_{\text {eff }} M$ and $R$ for UV spectrographs and telescopes. The product $A_{\text {eff }} M R$ has improved at a rate equivalent to a doubling every 16 months. An extrapolation of this trend to the immediate post-HST era could be a $4.6 \mathrm{M}$ diameter telescope with a total telescope plus spectrograph optical efficiency of $5 \%$, a detector quantum efficiency of $60 \%$ and a capability of recording a spectrum with $R=200,000$ over a bandpass of $600 \AA$.

\section{Secondary Parameters}

There are a number of secondary parameters that are sometimes overlooked, but which nevertheless have a bearing on how well we can meet diverse scientific demands. First, there is the equivalent background flux $F_{\mathrm{bkg}}$, which may or 
may not be uniform with wavelength or observing conditions. Even if $F_{\mathrm{bkg}}$ is known with good precision, the increased statistical fluctuations will still make the $S / N$ degrade by a factor $\sqrt{1+\left(F_{\mathrm{bkg}} / F_{\text {source }}\right)}$. Next, there are two important parameters that arise from the choice of the detector: (1) the maximum attainable $S / N$, which is limited by how well one can calibrate sensitivity variations, and (2) the flux above which a detector becomes unacceptably nonlinear or is at risk of being damaged. Finally, there may be scattered light, which arises from either imperfections in the gratings or deficiencies in the spectrograph's baffling scheme. Scattered light increases $F_{\mathrm{bkg}}$ and can be particularly bothersome if it is difficult to characterize.

\section{The Importance of Achieving High Resolution}

Interstellar absorptions often consist of line complexes that have narrow velocity structures with small separations. If one simply wants to measure the total column density, it is often satisfactory to have resolutions that are far inferior to those needed to resolve the individual features. This obviously applies to very weak, unsaturated features, but Jenkins $(1986,1996)$ has shown that even collections of moderately saturated features can be analyzed if two or more transitions of differing strength are available. While this may be true, it is sometimes necessary to recognize the existence of sharp lines arising from cold material with a small velocity dispersion that may be hidden among other, broader lines. Tripp $\&$ Bowen (2003) illustrate the loss of such information at $R=20,000$. Also, it may be important to recognize differences in the velocity structures between different species within a multiphase medium, as opposed to what may be seen for a homogeneous mixture (Tripp, Savage, \& Jenkins 2000). Sometimes, significant insights arise from small kinematical differences of different species (Jenkins $\&$ Peimbert 1997). Small shifts in $\lambda$ arise from important atomic and molecular processes, such as fine-structure excitations (Jenkins \& Tripp 2001), isotope shifts (Jenkins \& Wallerstein 1999), and rotational excitations of non-hydride molecules (Lambert et al. 1994; Lambert, Sheffer, \& Federman 1995).

\section{Negative Consequences that Might Arise when $R$ is Increased}

There are a number of considerations that, superficially, might be used as arguments against increasing $R: \mathbf{1}$. The detector can accommodate no more than some fixed value of $M$, so an increase in $R$ means that the wavelength coverage in a single exposure must be sacrificed. This is true for STIS on HST: for instance, one must make a choice between $R=120,000$ and $\Delta \lambda=200 \AA$ (E140H) or $R=46,000$ and $\Delta \lambda=600 \AA$ (E140M). However, the original STIS proposal in 1985 specified a far-UV detector with 4 times as many pixels as the one finally adopted for the instrument (to reduce development risks). It is therefore not outlandish to propose that after 2010 a $\Delta \lambda$ of about $800 \AA$ with $R=120,000$ is a realistic goal. 2. As $R$ increases, $A_{\text {eff }}$ must decrease, thereby compromising research on faint objects. Again, this is true for STIS on HST: modes with successively higher $R$ have lower $A_{\text {eff }}$. However, the product $A_{\text {eff }} M$ is an order of magnitude higher in the high resolution modes. 3. The increased detector 
area per unit $\Delta \lambda$ causes $F_{\mathrm{bkg}}$ to increase, thus further limiting research on faint targets. There are ways to reduce the detector background without resorting to exotic technologies: (1) cool the detector and use a photocathode with a high work function to reduce the spontaneous emission rate, (2) use materials within the detector that have very low concentrations of naturally radioactive elements, and (3) use a detector that can sense individual photoevents with a good timing accuracy and then build an anticoincidence shield so that events caused by energetic charged particles can be vetoed. 4. A high-resolution spectrograph places unacceptably large demands on the rest of the facility (e.g., small telescope pointspread function, tight pointing requirements, etc.). This is a moderately difficult issue. An echelle grating operating in a Littrow configuration has a sensitivity to angular deviations given by $1 / R=d \lambda / \lambda=\cot \alpha d \alpha / 2$, where $\alpha$ is the angle of incidence. Deviations in $\alpha$ are magnified by the ratio of the main telescope's focal length to that of the collimator, which favors a large diameter collimator (and grating) to increase $R$. A possible solution that virtually eliminates the angular dependence is to use a UV interferometer (Harlander, Reynolds, \& Roesler 1992; Stephan et al. 2001), perhaps working in conjunction with a conventional spectrograph (Edelstein \& Miller 1999; Erskine 2003). However, such an arrangement will probably lower the overall optical efficiency.

\section{References}

Edelstein, J., \& Miller, T. N. 1999, in ASP Conf. Ser. 164, Ultraviolet-Optical Space Astronomy beyond HST, ed. J. A. Morse, J. M. Shull \& A. L. Kinney (San Francisco: ASP), 333

Erskine, D. J. 2003, PASP, 115, 255

Harlander, J., Reynolds, R. J., \& Roesler, F. L. 1992, ApJ, 396, 730

Jenkins, E. B. 1986, ApJ, 304, 739

- 1996, ApJ, 471, 292

- 1999, in Chemical Evolution from Zero to High Redshift, ed. J. R. Walsh \& M. R. Rosa (Berlin: Springer), p. 280

Jenkins, E. B., \& Peimbert, A. 1997, ApJ, 477, 265

Jenkins, E. B., \& Tripp, T. M. 2001, ApJS, 137, 297

Jenkins, E. B., \& Wallerstein, G. 1999, in ASP Conf. Ser. 164, Ultraviolet-Optical Space Astronomy beyond HST, ed. J. A. Morse, J. M. Shull \& A. L. Kinney (San Francisco: ASP), 118

Lambert, D. L., Sheffer, Y., \& Federman, S. R. 1995, ApJ, 438, 740

Lambert, D. L., Sheffer, Y., Gilliland, R. L., \& Federman, S. R. 1994, ApJ, 420, 756

Stephan, S. G., Chakrabarti, S., Vickers, J., \& Cook, T. 2001, ApJ, 559, 491

Tripp, T. M., \& Bowen, D. V. 2003, in ASP Conf. Ser. 291, Hubble's Science Legacy: Future Optical/Ultraviolet Astronomy from Space, ed. K. R. Sembach, J. C. Blades, G. D. Illingworth \& R. C. Kennicutt (San Francisco: ASP), 407

Tripp, T. M., Savage, B. D., \& Jenkins, E. B. 2000, ApJ, 534, L1 